\title{
Micro mobility marketing: Two cases on location-based supermarket shopping trolleys
}

Received (in revised form): 15th November, 2007

\begin{abstract}
Jens Henrik Hosbond
is a PhD student in Information Systems at the Department of Computer Science at Aalborg University. In his PhD study, he is working on mobile software development (MSD) and more specifically on questions such as: What characterises it? What are the issues within MSD and why? And how can MSD practice be improved? Other research interests are innovation and agility on software development. He can be reached at joenne@cs.aau.dk.
\end{abstract}

\begin{abstract}
Mikael B. Skov
is an associate professor in the Human-Computer Interaction Lab at Aalborg University and holds a PhD in Human-Computer Interaction from 2002. His research interest is centred around mobile and pervasive computing, with a particular focus on understanding and extending existing approaches to evaluating mobile systems and how to utilise location-based services.
\end{abstract}

Keywords mobile marketing, location-based services, micro mobility, supermarkets, shopping trolleys, mobile consumers

\begin{abstract}
Mobile marketing refers to marketing of services or goods using mobile technology and mobile marketing holds potentially great economical opportunities. Traditionally, mobile marketing has been viewed as mobility in the large taking place virtually anywhere, anytime. Further, research shows considerable number of studies on push-based SMS mobile marketing campaigns. This paper explores a related yet different form of mobile marketing namely micro mobility marketing. Micro mobility marketing denotes mobility in the small, meaning that promotion of goods takes place within a circumscribed location, in our case a medium-sized retail supermarket. Two prototypes based on push and pull marketing strategies are implemented and evaluated. Taking outset in a synthesis of central issues in contemporary research on mobile marketing, we discuss their role in micro mobility marketing to point to similarities and differences between micro mobility marketing and the traditional mobile marketing in the large. Finally, we suggest areas for further study.

Journal of Targeting, Measurement and Analysis for Marketing (2007) 16, 68-77. doi:10.1057/palgrave.jt.5750058; published online 24 December 2007
\end{abstract}

\section{INTRODUCTION}

Mobile technologies have become more and more widespread as hardware technology has become less expensive and network infrastructure more accessible. Over the last decade, several innovative mobile services have grown enormously, such as short message service (SMS).

\footnotetext{
Correspondence: Mikael B. Skov, Department of Computer Science, Aalborg University, Selma Lagerlofs Vej 300, Aalborg East DK-9220, Denmark.

Tel: +45 9635 8922;

Fax: +459635 9798;

E-mail: dubois@cs.aau.dk
}

The development of mobile technologies is visible through the penetration of mobile phones over the last few years; in 1997 about 215 million people used mobile phones, by 2001 this figure had grown to 961 million people and in 2003 more than 1.16 billion people used mobile phones. ${ }^{1}$ Currently, mobile technologies converge, providing new functionality and opportunities as well as new innovative areas for business development. As immediate consequences, mobile technology development and innovation has received more and more attention from both academia and industry. 
Such advances in mobile technologies and supporting network infrastructures create opportunities for mobile marketing. Simplified, mobile marketing refers to marketing of services or goods using mobile technology. Examples of current mobile marketing often involve pushing information on promotion or discounting through SMSs to mobile phones of potential consumers in certain situations. Typically, the overall idea is that information can be better directed towards potential consumers by making the marketing location-sensitive or location-based. Location-based marketing can be highly relevant for local advertising; for example receiving information on train departures or arrivals at a train station. ${ }^{2}$

When attempting to provide location-based services, physical context becomes vital. Marketing producers need to understand the physical context in which the consumer is located in order to provide relevant and timely promotion. As an example, pushing information on discounted tickets to travellers in a train station is only relevant and timely if the consumer is able to utilise the discounted offer. Several years of research on mobile technologies have shown that we need to distinguish between different kinds of mobility. ${ }^{3}$ Mobility is highly defined by the way that a consumer is mobile and mobile marketing initiatives would benefit from a better understanding of the consumer mobility.

This paper promotes a particular focus on mobile marketing namely marketing in the small. We denote this as micro mobility marketing. First, we introduce related work and outline our understanding of micro mobility marketing. Secondly, we illustrate micro mobility marketing through two cases of location-based services in retail supermarkets. Thirdly, we discuss our findings in terms of known issues of mobile marketing.

\section{MOBILE MARKETING}

Mobile marketing originates from research on mobile commerce and marketing but still requires exploration in order to reach the necessary level of academic conceptualisation. Mobile commerce has attracted considerable attention and still does (see eg Senn, ${ }^{4}$ Varshney et al., ${ }^{5}$ Siau et al. ${ }^{6}$ and Varshney and Vetter ${ }^{7}$ ). M-commerce has broadly been defined as 'the use of mobile (handheld) devices to communicate and conduct transactions through public and private networks'. ${ }^{8}$

Mobile marketing is a relatively new field of interest. ${ }^{9}$ Mobile marketing possesses great economical potential with an estimated spending of $\$ 428.4 \mathrm{~m}$ in 2007 in the US alone. ${ }^{10}$ In this paper, we adopt the following conceptualisation where mobile marketing is defined as 'using interactive media wireless media to provide customers with time and location sensitive, personalized information that promotes goods, services and ideas, thereby generating value for all stakeholders'. ${ }^{2}$

Research within mobile marketing has been dominated by studies on push-based SMS marketing. ${ }^{9}$ In push-based mobile marketing, advertisements get pushed to the mobile device of the consumer. Whether the user has accepted or not to receive these advertisements differs. This relates to permission-marketing, which is based on obtaining consent from the recipient in order to avoid spamming consumers that generally only leads to negative consumer perceptions of advertising. Examples of push-based SMS studies are Barwise and Strong ${ }^{11}$ that explore the effectiveness of SMS marketing campaigns in reaching young adults in the UK. Heinonen and Strandvik ${ }^{12}$ study consumer responsiveness to mobile marketing based on SMS advertising. Tsang et al. ${ }^{13}$ and Carroll et al. ${ }^{14}$ explore consumer attitude towards SMS-based mobile advertising and finally Dickinger et al. ${ }^{2}$ and Scharl et al. ${ }^{15}$ explore the potential of SMS-based marketing where both studies provide pointers as to what the success factors of implementing SMS marketing campaigns are. On the other hand, we have seen rather limited research on mobile marketing based on pull-based marketing strategies although examples of studies exist, for example Okazaki. ${ }^{16}$ In pull-based mobile marketing, the consumer is in complete control of the ads he/she receives.

Common for these research studies is the fundamental focus on mobility in the large. Often these studies do not distinguish between different 
levels of mobility and what implications this may have on the conditions for the mobile marketing solution and the resulting consumer use and perception. By mobility in the large we mean mobility without any physical boundaries. In other words, the interaction between the end user and the mobile marketing medium can take place anywhere and anytime.

In this paper, we explore mobile marketing in the small. We adopt the term micro mobility inspired by the work by Luff and Heath as a descriptor for the type of bounded spatial mobility characterising two mobile marketing prototypes for retail supermarkets. ${ }^{17}$ Mobility has been discussed and investigated in related research, that is, Kakihara and Sørensen ${ }^{18}$ present an overview of the various conceptualisations on mobility. They suggest spatiality (location), temporality (time) and contextuality (in what way, under what circumstances and towards which actors) as three different but related dimensions of mobility. Also, Luff and Heath ${ }^{17}$ proposed micro mobility as being 'the way in which an artefact can by mobilised and manipulated for various purposed around a relatively circumscribed, or "at hand", domain'. We adopt the latter definition for mobile marketing in the small namely micro mobility marketing.

\section{MICRO MOBILITY MARKETING: TWO CASES}

This section presents two different but related mobile marketing prototypes that serve as illustrative examples of micro mobility marketing. Both cases utilise mobile technologies that support the promotion of goods in retail supermarkets. Micro mobility is constrained to the activities taking place within a relatively circumscribed domain.

The prototypes were originally designed and implemented in two separate research projects and while sharing a number of similarities, they differ in marketing strategies and how they were evaluated through a series of consumer testing. But most specifically, the two cases are different in terms of mobile marketing according to their implementation of push and pull of information. As discussed above, issues related to permission and disturbance are important when producers promote services or products through mobile marketing. Fundamentally, information can either be pushed to the mobile devices or the consumer can pull information. Our first case primarily illustrates a micro mobility marketing strategy using information pull while the other case defines a strategy using information push. We describe the first case that implements mobile marketing through promotion and display of goods on an interactive interface mounted on a shopping trolley. Goods are promoted from a predefined shopping list. Afterwards, we describe the second case where mobile marketing is based on consumer purchase history and goods are promoted and displayed according to expected preferences of the shopper. This prototype also utilises an interactive touch interface mounted on a shopping trolley.

\section{Marketing context: A medium-sized retail supermarket}

The physical context for both cases is a branch of the supermarket chain føtex located in Aalborg East, Denmark. This provides items primarily within food, but also electronics, clothes, etc. The supermarket is app. 3,500 square meters and contains 12 check-out points.

Prior to the design and implementation of the prototypes, we conducted a number of field studies in føtex in an attempt to better understand mobility in this context. This was done by observing a number of shoppers while shopping in the supermarket. We observed how consumers would move around in the store and what products they looked for and put into their shopping trolley. Also, we observed the consumers while trying to locate different items from their shopping list. The findings of these observations formed the design of the two prototypes for micro mobility marketing.

\section{Case A: Context-Aware Shopping Trolley (CAST)}

Our first case outlines the Context-Aware Shopping Trolley (CAST). CAST supports shopping by promoting products in the supermarket and through guidance on how to 

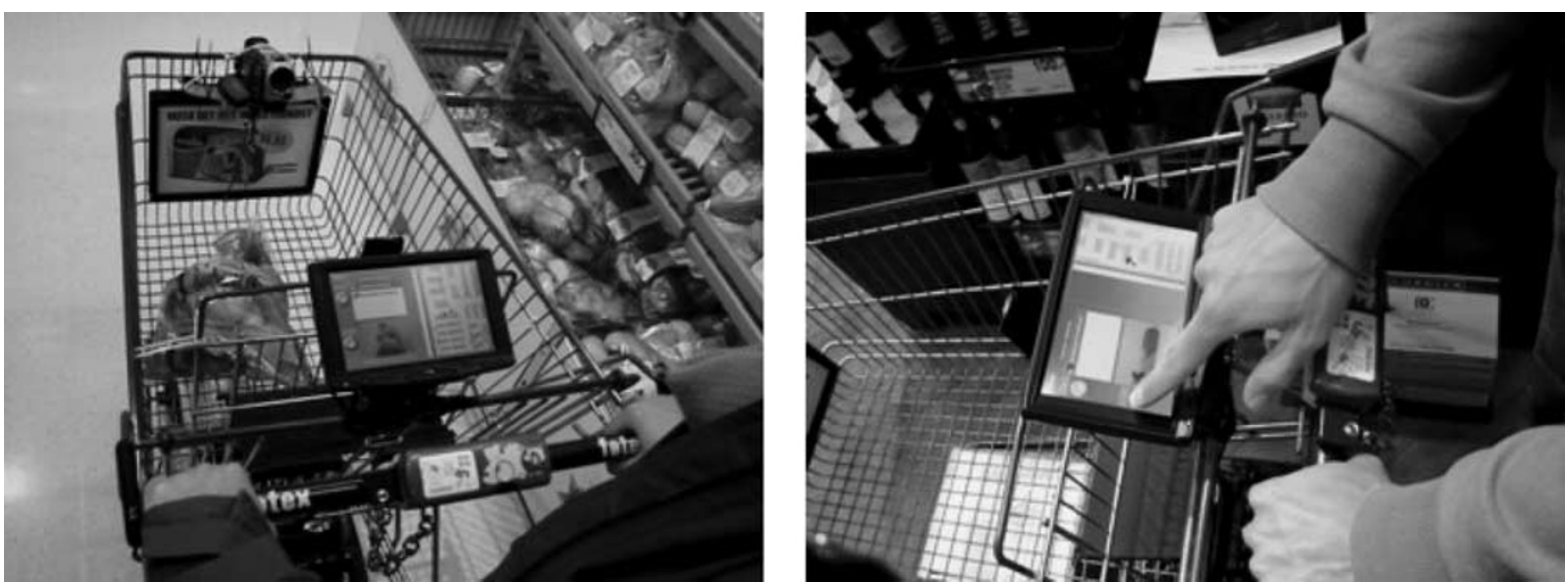

Figure 1: Illustrations of CAST in use. The visual appearance of items from the shopping list is shown on the touch screen and the location of products in close proximity of the consumer is visualised

locate these products. Products are visually displayed on the trolley-mounted screen with a photographic picture and the product's physical location in the supermarket (see Figure 1). Products will be chosen for the display from a specified shopping list. CAST implements a pullbased strategy where shoppers can request more information on products or on additional products than specified in the shopping list.

From our initial empirical investigation, we found that supermarket shopping often involves specified shopping from a shopping list where consumers more or less have pre-selected items to be bought. Hence, the primary task of the consumer was to locate and buy items specified in the shopping list. The list provides a reference for the consumer where he/she can see what needs to be done before their task is considered complete. In order to support the consumer in this way, we update the list when an item is collected and placed in the trolley. The system also calculates the sub-total for all collected items.

For grocery marketing, we sought to utilise location in several ways by integrating the location of consumers, products and shelves, and also the spatial relations between them. A map offers the consumer a resource for physical orientation by outlining a blueprint of føtex illustrating the layout of the shelves and aisles. The items on the shopping list are represented with icons corresponding to those displayed in the shopping list to aid recognition. Finally, the position of the consumer is also indicated on the map. As the consumer moves around, the map is automatically updated and the shopping list reorders according to product proximity.

In addition to supporting consumer location awareness, CAST promotes products in close proximity. Getting close to a specific product, the system sends an audio alert through the user's headset and displays a list of nearby products. By tapping an item in the screen, its position is illustrated on the map. In this state, only products that are considered nearby (ie listed in the popup) are shown on the map; all other icons are temporarily removed to reduce complexity. To achieve this functionality, each product was associated with a rectangular area of the shop floor where the product is considered nearby (Figure 2).

CAST targets the supermarket products to the consumer in multiple ways: (1) location of products, (2) visual appearance of products, (3) existence of relations between products and (4) information about products. The basic functionality combines the first two dimensions by providing a photograph of nearby products. CAST supports the consumer's knowledge of the existence of and relations between objects by showing products similar and related to those on the shopping list. The consumer can select an item from the shopping list to view a list of 

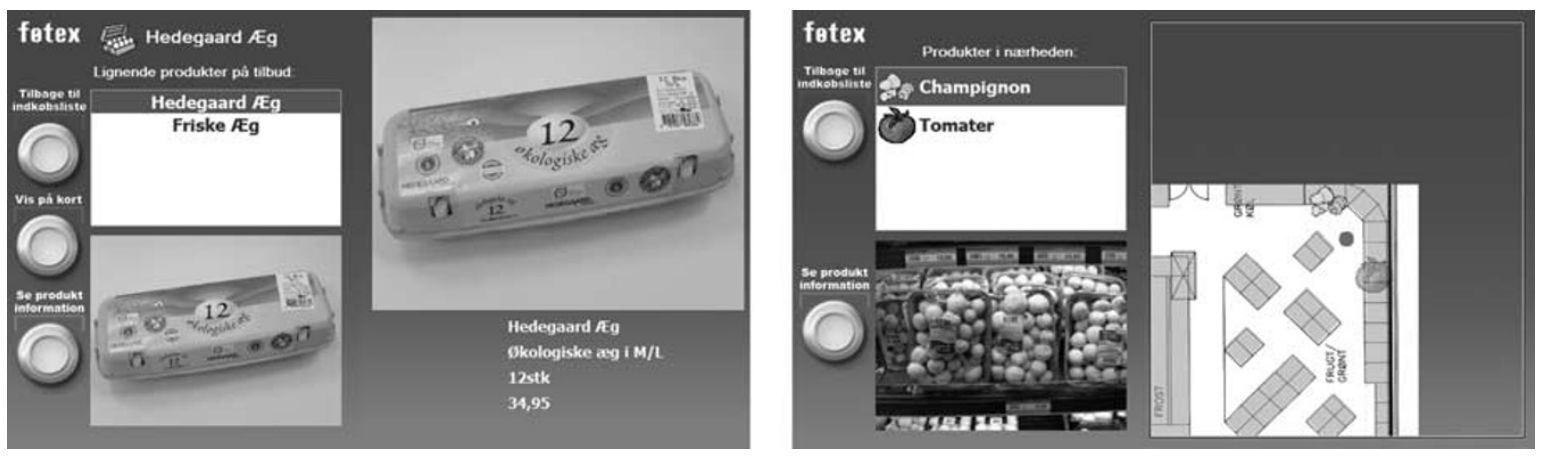

Figure 2: Screen shots from CAST where products are illustrated through images and their physical location in the supermarket

alternative products. The user can select an alternative product, displaying its picture, and view more detailed information related to the product. This functionality has been included for multiple purposes - it facilitates more traditional 'information pull' interaction with CAST and thus allows the system to be useful in cases where its own recommendations are deemed otherwise.

CAST provides descriptive information about products by allowing the consumer to view information about any product. This information is displayed in a panel that pops up from the bottom of the screen, and covers the map. This panel can be called from either the nearby products popup, or the alternative products popup. We note that there are some abstraction challenges inherent to designing a shopping aid system like CAST with regard to products. CAST implements two levels of product generalisation - specific products (eg Gillette Pure Gel) and product groups (eg Shaving Gel). In the event that a product group is entered in the shopping list, all the products in that group are displayed when they are nearby. For instance where 'Bacon' is an item on the shopping list, and the shopper is near the bacon products, he/she is shown the various types of bacon on offer.

\section{Case B: Personal Shopping Assistance (PSA)}

Our second case outlines the Personal Shopping Assistant (PSA). PSA is a mobile prototype and supports the shopping experience in supermarkets by continuously monitoring consumer behaviour to enable the pushing of product ads. More specifically, the primary service delivered by the system is to deliver relevant product information to customers based on personal preferences when and where the need arises. Specifically, relevant advertisements are dynamically and visually displayed for each customer as she moves around in the store.

We have designed PSA from a three-folded marketing perspective. First and foremost, the system is designed to create improved shopping experiences for consumers as PSA, continuously during shopping, provides shoppers with tailored promotions and marketing of supermarket products, that is, groceries. Secondly, while the primary focus is on the consumer, it is expected that advertisements presented at the right time, the right place and to the right consumer will create increased sales. Hence, the system also carries a strong business incentive for implementation. Thirdly, PSA provides potentially a platform for manufacturer advertisements.

In the following, we will illustrate PSA through a simple use scenario. A consumer needs new groceries. She logs in to her personal web shopping account from her PC, (illustrated in Figure 3). The web account includes a range of features, with the central ones being: altering her personal profile, managing (create/edit/delete) shopping lists and view historic data of her previous purchases from the supermarket. Having created a new shopping list, she drives to the supermarket. Arriving at the supermarket she takes a shopping trolley on which an ultra mobile PC (UMPC) together with a touch-screen and an attached bar code reader has been mounted. She 


\section{PAYWAY}

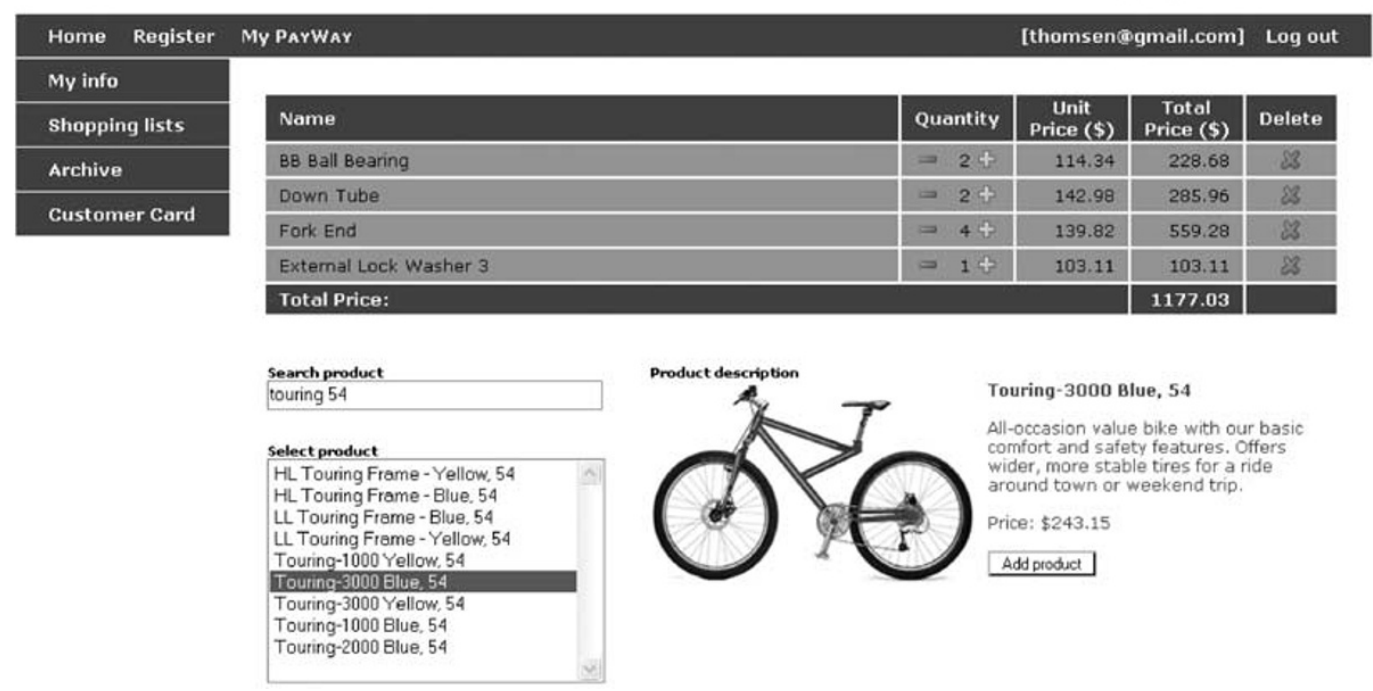

Figure 3: Web user interface for a given consumer's shopping account

swipes her personal PSA plastic shopping card through the barcode reader on the UMPC and her profile is instantly and wirelessly validated by the PSA system running in the supermarket. Upon personal validation, the most recent shopping list is displayed on the screen of the UMPC (illustrated in Figure 4).

The displayed shopping list provides the consumer with a checklist on the groceries to purchase. Besides the view of the shopping list, the prototype also provides secondary features such as a graphical display of the store plan, a price check function and a cookbook that suggests recipes based on what has been put into the shopping trolley.

Taking the first product on the shopping list from the shelf and scanning the product's barcode with the bar code reader attached to the UMPC, the PSA system instantly locates the position of the shopping trolley. From the location of the shopping trolley, the PSA system automatically displays advertisements for products in close proximity. The display of advertisements is not pre-defined, but is dynamically selected at the back-end of the system by analysing the historical data on the customer's previous purchases. The advertised product should therefore appeal to the

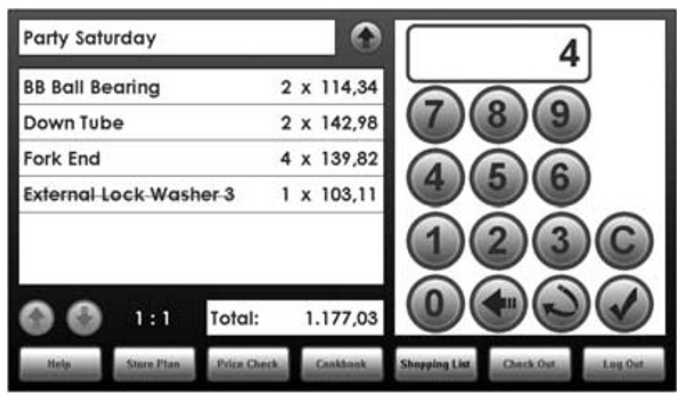

Figure 4: UMPC user interface showing the most recent shopping list

customer as they become meaningful in the context that the customer is now in. That is, advertisements are displayed at the right place, at the right time and to the right customer. When a product has been put into the trolley, the item is stroked through on the shopping list, indicating that it has already been picked up. The shopping list is edited as new products not initially on the shopping list are added to the trolley or when an item is discarded. As products are added, items on the shopping list are checked and a total is calculated based on the items in the shopping trolley. At checkout, the items are paid for and the PSA system adds the items to the personal archive of previous purchases. 


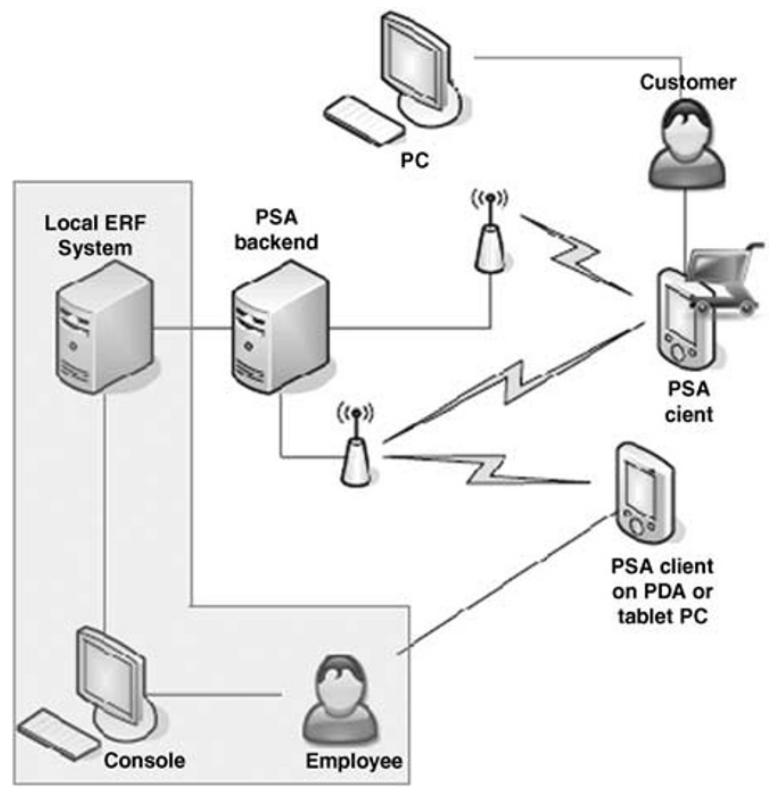

Figure 5: An overview of the PSA system architecture. The grey area illustrates the existing IT infrastructure in the supermarket

Data mining techniques were used for analysing customer patterns when shopping; and stored procedures were extensively used for optimising performance for accessing, creating, updating and deleting product and advertisement information in the back-end. The implementation has involved development of various software components all with the ability to integrate with existing enterprise IT in a supermarket. A complete overview of the different parts of the system and how they are connected is depicted in Figure 5.

\section{DISCUSSION}

We have suggested micro mobility marketing as a way of defining the mobile marketing in the small. We based our understanding of micro mobility marketing on the definition of mobile marketing, ${ }^{2}$ combined with a definition on mobility in the small. ${ }^{17}$ We presented two mobile marketing prototypes that served as illustrative examples of marketing in the small. Both cases utilised mobile technologies to support marketing of goods in a retail supermarket. Micro mobility was constrained to activities taking place within a relatively circumscribed domain. The two prototypes utilised different marketing strategies enabling pull- and push-based interaction. Both strategies, however, sought to facilitate mobile marketing in the small by promoting supermarket products on the screen based upon consumer interaction and movement in the store. Both prototypes were tested in a number of empirical evaluations with the objective of assessing the potential of mobile marketing in supermarkets.

Two issues seemed to strongly characterise the investigated domain of micro mobility marketing. First, the location exhibits clear boundaries where it is clear when shoppers are inside the supermarket and when they are outside. The location is relatively accessible and is circumscribed as defined by micro mobility. Second, the task is relatively clear namely to find and buy a number of products. Hence all shoppers shared the same objective, but they may have had different strategies when completing the task. Some shoppers have a pre-specified shopping list that strictly defines their task while other shoppers pursue a more browse-like strategy where the activity in itself identifies the products more dynamically. But from an overall perspective, their basic task can be considered the same. In the following, we will discuss the two mobile marketing prototypes in terms of key issues of mobile marketing namely permission and disturbance, content and context and user experience and usability. This is not meant as an exhaustive list, but merely an important list of relevant issues.

\section{Mobile marketing: Permission and disturbance}

Mobile marketing inherently adopts challenges related to the user - mobile device interaction. As providers of marketing and promotion seek to sell goods or services, they need to capture consumer intention through the mobile device. ${ }^{19}$ Two key issues regarding capturing consumer attention in mobile marketing are permission and disturbance.

Permission-based marketing is a nontrivial concern in mobile marketing. ${ }^{19-21}$ How do the marketing providers obtain consumer consent to send ads? One approach is to ensure a reward 
scheme for the consumer, that is, access to free extra services. In our two cases, the role of permission differs. First, in the CAST prototype the permission to send additional product information (ads) to the customer is explicitly given the moment he/she specifically requests related product information. In the PSA prototype, customer permission is implicitly given the moment the customer signs up for a personal account. Tentative results from the empirical evaluations indicate that our consumers did not find this to be a severe problem. By entering the supermarket and using either of the two prototypes, the consumers indicated that they had implicitly given consent to receive ads about supermarket products. We would have to stress that only ads relevant to the context and task were promoted during our evaluations. The task is to shop in a supermarket and the location is bounded by the physical structures of the building. Knowing the location and the task makes it possible to provide value-adding information fitting to the context in which the user resides. Finally, we would like to stress that the two prototypes could be adopted for competing marketing strategies between different manufacturers that could affect consumer acceptance as several of our consumers indicated strong bias towards various brands.

Another important issue related to the capturing of consumer attention is disturbance (and acceptance). These issues primarily concern the assessment of maximum consumer disturbance levels with marketing content while still being accepted by the consumer. ${ }^{12}$ In our two cases, disturbance and acceptance relate to the consumer's perception of (1) the frequency with which the prototypes push product information onto the mobile device (ie, the UMPC) and (2) the relevance of the advertisements. Our PSA consumer was disturbed each time she came in proximity of a product on her shopping list or when approaching products fitting her. The level of disturbance was therefore tightly bounded to the items on the shopping list. In addition, it is important to note that the content 'disturbing' the customer is personalised and tailor made to fit the specific user. One implication of this may be that the accepted level of disturbance for a user may be higher than usual. The frequency of disturbance was actually tested for the CAST consumers. Archetypes of customers tested the prototype in the supermarket and as the users moved around in the supermarket, the level of interruption was deliberately increased. Customers were notified of related products by audio notifications. In the test we received no negative feedback on the level of disturbance. Instead, users perceived the disturbance as helpful and valueadding in the context of navigating and shopping in the supermarket. Several of our consumers indicated, however, that the novel effect of simply using the system was positive and rewarding.

\section{Mobile marketing: Content and context}

While permission and disturbance constitute two highly relevant issues for mobile marketing, providers of marketing campaigns must provide relevant and interesting content to the mobile consumer and they have to consider the context of the marketing campaign as well.

The content of mobile marketing ads is highly relevant and necessary in ensuring customer acceptance and responsiveness to the presented marketing campaign. ${ }^{1,12,22}$ Based in micro mobility marketing where the primary user task was known, this issue becomes much less problematic for our consumers. The CAST prototype provided relevant information through immediate on-screen display of products related to the items in the shopping list and to the location of the trolley. In the PSA prototype, an extra dimension of content relevancy was added namely personalisation of content. The personalisation of content is based on three things: (1) the current location of the shopping trolley, (2) historic data on the customer's previous purchases and (3) items on the shopping list. Dwelling on the two approaches for delivering relevant content, the PSA prototype clearly provides the most advanced scheme for serving relevant content, hence, also the one most likely to experience success. Thus, our initial findings showed that the predefined consumer task in supermarkets tailored the content of the 
marketing ads in the two prototypes, and none of our evaluation participants questioned the relevance of the content.

Context defines the effectiveness of the mobile marketing campaign as it depends on the ability to provide the consumer with adverts at the right time and at the right place. ${ }^{22}$ Given the welldefined and delimited spatial location in our cases, we identified no major concerns related to context relevancy. We found that the circumscribed domain characteristics of micro mobility marketing solved some of the fundamental challenges of mobile marketing. In particular, the physical location of the marketing campaign is well known and well defined; shopping is taking place only in the store. But, on the other hand, location is not only the same location within the store as the supermarket was divided into separate segments of items. Temporal aspects were only limited integrated into the prototypes. Both prototypes considered when to promote goods or campaigns on the interactive screen while shopping in the supermarket. Our cases, however, did not integrate additional issues related to temporal aspects.

\section{Mobile marketing: User experience and usability}

As mobile marketing utilises emerging and contemporary information technologies, aspects of user experience and usability become crucial as consumers interact with these technologies.

The perceived user experience of the mobile marketing solution has a direct effect on its success. ${ }^{22}$ User experience is often difficult and challenging to measure and we only have limited empirical evidence on user experience for our two prototypes. Our users, however, were generally quite positive towards the idea of interacting with a mobile interactive technology mounted on the shopping trolley. Most stated that this in itself contributed to a higher shopping experience and thus had a positive impact on the user experience. Furthermore, several consumers indicated that they were very or somewhat positive towards receiving ads or campaign information on such mobile marketing devices as it could potentially improve their shopping activity with respect to either saving money or locating new exciting products. Hence, the added value of the two prototypes was rather clear. But the user experience was difficult to fully examine as several of our test users stated that simply just using new interactive media during shopping was exciting and rewarding.

The consumer needs to perceive the mobile marketing channel as usable and not intervening with important matters. It has to be immediate. ${ }^{22}$ Like some of the other characteristics of mobile marketing, usability and immediacy were generally high in the two cases as the promotion and campaign information implicitly or explicitly supported the task at hand for the shoppers. In CAST, the mobile marketing of items supported the shoppers in identifying and locating the items within the supermarket. Our study showed that consumers had difficulties in recognising and locating products in the supermarket and the CAST prototype assisted the shoppers in solving these challenges. On the other hand, several of our test consumers had minor difficulties when interacting with the mobile prototype, for example the accuracy of the map proved to be rather important as the users attempted to create mental models of the map.

\section{Mobile marketing: Micro mobility marketing}

Our work on mobile marketing in the small seemed to solve (or at least address) some of the fundamental issues of mobile marketing. Especially, issues concerning permission and content regarding mobile ad campaigns were less problematic for micro mobility marketing as the consumers in our study had already accepted the engagement. Also, relevancy in terms of targeting ads to relevant consumers was substantially achieved through knowing user task and assignment and also through profiling of the consumer. Thus, our findings indicate that micro mobility marketing can support providers of mobile marketing campaigns with valuable guidance on specifying consumer behaviour in terms of tasks and the intended context of use.

We are, however, aware that several other domains could provide more difficult challenges 
in defining user tasks and contexts and thus, it becomes increasingly harder to define the scope of micro mobility marketing.

\section{CONCLUSION}

Mobile marketing refers to marketing of services or goods using mobile technology and mobile marketing holds potentially great economical opportunities with an estimated spending of $\$ 428.4 \mathrm{~m}$ in 2007 in the US alone. ${ }^{10}$ We have tried to contribute to a more nuanced understanding of mobile marketing by suggesting a focus on mobility marketing in the small. We denote this micro mobility marketing. Micro mobility marketing is different from traditional large-scale mobile marketing campaigns in that it is characterised by a spatially bounded area in which it is to be carried out. We exemplified micro mobility marketing by developing two mobile marketing prototypes for a medium-sized Danish supermarket.

The two prototypes are based on either a push-based or a pull-based marketing strategy. By relating the prototypes and their user evaluations to central concerns synthesised from the research body of mobile marketing, we find that in micro mobility marketing some of the issues traditionally found in the mobile marketing literature become almost irrelevant, significantly increasing the chances of a successful marketing campaign. Micro mobility marketing has shown potential, but needs further conceptualisation and in-depth user studies need to be conducted in order to further validate and explore this new form of mobile marketing.

\section{Acknowledgments}

The work in this paper received financial support from the Danish Research Agency (grant no. 2106-04-0022). We especially thank all the participating test subjects. Furthermore, we wish to thank several anonymous reviewers for comments on drafts of this paper.

\section{References}

1 Bauer, H. H., Barnes, S.J., Reichardt, T. and Neumann, M.M. (2005) 'Driving consumer acceptance of mobile marketing: A theoretical framework and empirical study', Journal of Electronic Commerce Research, Vol. 6, No. 3, pp. 181-192.
2 Dickinger, A., Haghirian, P., Murphy, J. and Scharl, A. (2004) 'An investigation and conceptual model of SMS marketing', Proceedings of the 37th Hawaii Conference on System Sciences, IEEE, Hawaii.

3 Kristoffersen, S. and Ljungberg, F. (2000) 'From stationary to mobile workin Braa, K., Sørensen, C. and Dahlbom, B. (eds), 'Planet Internet', Studentlitteratur, Lund, pp. 41-64.

4 Senn, J. (2000) 'The emergence of M-commerce', Computer IEEE Computer Magazine, Vol. 33, No. 12, pp. 148-150.

5 Varshney, U., Vetter, R.J. and Kalakota, R. (2000) 'Mobile commerce: A new frontier', IEEE Computer, Vol. 33, No. 10, pp. 32-38.

6 Siau, K., Lim, E. and Shen, Z. (2001) 'Mobile commerce: Promises, challenges, and research agenda', Journal of Database Management, Vol. 12, No. 3, pp. 4-13.

7 Varshney, U. and Vetter, R. (2002) 'Mobile commerce: Framework, applications and networking support', Mobile Networks and Applications, Vol. 7, No. 3, pp. 185-198.

8 Balasubramanian, S., Peterson, R. A. and Jarvenpaa, S.J. (2002) 'Exploring the implications of $\mathrm{M}$-commerce for markets and marketing', Journal of the Academy of Marketing Science, Vol. 30, No. 4, pp. 348-361.

9 Okazaki, S. (2005) 'New perspectives on M-commerce research', Journal of Electronic Commerce Research, Vol. 6, No. 3, pp. 160-164.

10 Elkin, N. (2005) 'Mobile marketing and M-commerce: Global spending and trends', Retrieved February 2005, from http:// www.emarketer.com/Report.aspx?mobile_mktg_feb05.

11 Barwise, P. and Strong, C. (2002) 'Permission-based mobile advertising', Journal of Interactive Marketing, Vol. 16, No. 1, pp. 14-24.

12 Heinonen, K. and Strandvik, T. (2003) 'Consumer responsiveness to mobile marketing', Proceedings of Stockholm Mobility Roundtable, Stockholm.

13 Tsang, M. M., Ho, S. and Liang, T. (2004) 'Consumer attitudes toward mobile advertising: An empirical study', International Journal of Electronic Commerce, Vol. 8, No. 3, pp. 65-78.

14 Carroll, A., Barnes, S. J. and Scornavacca, E. (2005) 'Consumers perceptions and attitudes towards SMS mobile marketing in New Zealand', Proceedings of International Conference on Mobile Business (ICMB), 2005, IEEE.

15 Scharl, A., Dickinger, A. and Murphy, J. (2005) 'Diffusion and success factors of mobile marketing', Electronic Commerce Research and Applications, Vol. 4, pp. 159-173.

16 Okazaki, S. (2004) 'How do Japanese consumers perceive wireless ads? A multivariate analysis', International Journal of Advertising, Vol. 23, pp. 429-454.

17 Luff, P. and Heath, C. (1998) 'Mobility in collaboration', Proceedings of the 1998 ACM Conference on Computer Supported Cooperative Work ACM Press, Seattle, Washington, United States.

18 Kakihara, M. and Sørensen, C. (2002) 'Mobility: An extended perspective', Proceedings of the 35th Hawaii International Conference on System Sciences, IEEE, Hawaii.

19 Barnes, S. J. and Scornavacca, E. (2004) 'Mobile marketing: The role of permission and acceptance', International Journal of Mobile Communications, Vol. 2, No. 2, pp. 128-139.

20 Godin, S. (1999) 'Permission Marketing', Simon \& Schuster, New York.

21 Kavassalis, P., Spyropoulou, N., Drossos, D., Mitrokostas, E., Gikas, G. and Hatzistamatiou, A. (2003) 'Mobile permission marketing: Framing the market inquiry', International Journal of Electronic Commerce, Vol. 8, No. 1, pp. 55-79.

22 Facchetti, A., Rangone, A., Renga, F.M. and Savoldelli, A. (2005) 'Mobile marketing: An analysis of key success factors and the European value chain', International Journal of Management and Decision Making, Vol. 6, No. 1, pp. 65-80. 